\title{
Postoperative Hypoparathyroidism and the Viability of the Parathyroid Glands During Thyroidectomy
}

\author{
Yong Bae $\mathrm{Ji}^{1} \cdot$ Chang Myeon Song ${ }^{1} \cdot$ Eui Suk Sung ${ }^{1} \cdot$ Jin Hyeok Jeong ${ }^{1} \cdot$ Chang Beom Lee ${ }^{2} \cdot$ Kyung Tae $^{1}$ \\ Departments of ${ }^{1}$ Otolaryngology-Head and Neck Surgery and ${ }^{2}$ Internal Medicine, Hanyang University College of Medicine, Seoul, Korea
}

Objectives. To prevent hypoparathyroidism after thyroidectomy, preservation of the parathyroid glands and their vascularity are essential. The aim of this study was to determine the association between postoperative parathyroid function and the viability of the parathyroid glands during thyroidectomy.

Methods. We prospectively analyzed 111 patients who underwent total thyroidectomy and in whom all 4 parathyroid glands were preserved in situ during the operation. The surgeons scored the viability of each parathyroid gland from 0 (normal) to 3 (severely compromised viability) based on its gross appearance and vascularity intraoperatively. The index of parathyroid viability score (IPVS) was defined as the sum of the viability scores of the 4 parathyroid glands. We evaluated the relationship between postoperative parathyroid function and IPVS.

Results. Transient hypoparathyroidism occurred in 25 patients (22.5\%), and permanent hypoparathyroidism in 4 patients (3.6\%). The IPVS were significantly different in the three groups: $2.87 \pm 1.46$ in the normal group, $3.68 \pm 1.41$ in the transient hypoparathyroidism group and $7.50 \pm 1.00$ in the permanent hypoparathyroidism group. The rates of transient hypoparathyroidism were $13.6 \%$ in patients with IPVS $0-2,23.8 \%$ in patients with IPVS $3-4$, and $42.9 \%$ in patients with IPVS 5-6. All the patients with IPVS of 7 or more had permanent hypoparathyroidism.

Conclusion. IPVS is correlated with the incidence of hypoparathyroidism. It could be a good quantitative indicator of the probability of hypoparathyroidism after thyroidectomy.

Keywords. Parathyroid Glands; Hypoparathyroidism; Hypocalcemia; Thyroidectomy

\section{INTRODUCTION}

Hypoparathyroidism is an important complication of thyroidectomy and results in hypocalcemia. The symptoms of hypocalcemia can be subtle, such as perioral or digital paresthesia, muscle cramp or anxiety, but sometimes it results in serious or lifethreatening symptoms such as tetany, altered mental status, seizure, cardiac arrhythmia, heart failure, and laryngospasm [1].

Although parathyroid function is restored in most cases, re-

\footnotetext{
- Received June 1, 2016

Revised July 5, 2016

Accepted July 8, 2016

- Corresponding author: KyungTae

Department of Otolaryngology-Head and Neck Surgery, Hanyang

University College of Medicine, 222 Wangsimni-ro, Seongdong-gu, Seoul 04763, Korea

Tel: +82-2-2290-8585, Fax: +82-2-2293-3335

E-mail: kytae@hanyang.ac.kr
}

covery is sometimes insufficient due to irreversible injury to the parathyroid glands. In such cases, lifelong supplementation of calcium is inevitable. Not only direct injury including excessive manipulation or cautery but also indirect vascular injury can result in hypoparathyroidism. Furthermore, inadvertent excision of the parathyroid glands can occur [2-5]. The incidence of transient hypoparathyroidism after total thyroidectomy has been reported as from $10 \%$ to $46 \%$ and that of permanent hypoparathyroidism as from less than $1 \%$ to as high as $10.6 \%$ [6-9].

To prevent hypoparathyroidism, preservation of the parathyroid glands in situ as well as avoidance of injury to their vascular pedicles is essential. Devascularized parathyroid glands can be recognized intraoperatively by inspection because they become dark brown or even black. Sometimes, devascularization is caused by arterial insufficiency, and the glands do not become discolored. In cases of arterial insufficiency, viability can be determined by observing active bleeding after making small inci-

Copyright @ 2017 by Korean Society of Otorhinolaryngology-Head and Neck Surgery.

This is an open-access article distributed under the terms of the Creative Commons Attribution Non-Commercial License (http://creativecommons.org/licenses/by-nc/4.0)

which permits unrestricted non-commercial use, distribution, and reproduction in any medium, provided the original work is properly cited. 
sions in the parathyroid glands [10]. Any parathyroid gland that looks likely to be totally devascularized can be removed during the operation and autotransplanted into well-vascularized muscles such as the sternocleidomastoid muscle or the forearm muscles [11]. The thyroid gland and central neck dissection (CND) specimens excised should also be examined carefully to discover any inadvertently removed parathyroid glands, and these should be autotransplanted [2].

To minimize the morbidity and complications of hypocalcemia, early prediction of parathyroid function and immediate management is necessary. To predict postoperative parathyroid function, most previous studies analyzed postoperative serum levels of parathyroid hormone (PTH) or calcium immediately after the operation or within several hours [12-15]. Experienced thyroid surgeons can also estimate postoperative parathyroid function empirically by considering the number of parathyroid glands preserved in situ and their appearance. However, there has been little investigation of the correlation between postoperative parathyroid function and intraoperative parathyroid findings, namely their appearance and vascularity, although some studies have reported a correlation between the number of preserved parathyroid glands and postoperative parathyroid function $[8,16,17]$. We supposed that if the intraoperative viability of parathyroid glands during thyroidectomy was correlated with postoperative parathyroid function, the former could be used to quantitatively predict the probability of postoperative hypoparathyroidism. It might also be helpful in deciding about parathyroid autotransplantation and calcium replacement therapy.

The aim of this study was to determine the association between postoperative parathyroid function and the viability of the parathyroid glands based on the intraoperative gross findings and vascularity as evaluated by the surgeons.

\section{MATERIALS AND METHODS}

\section{Patients}

This study was approved by the Institutional Review Board of Hanyang University Hospital, Seoul, Korea (HYUH 2014-04019) and written informed consent was obtained from each pa-

\section{H I G H L I G G H T S}

- The viability of the parathyroid glands during thyroidectomy affects postoperative hypoparathyroidism.

- The viabilities of all 4 glands were prospectively evaluated by their visual grading from 0 (normal) to 3 (severely impaired).

- The scores were frequently high in patients with transient and permanent hypoparathyroidism.

- The visual scoring might be a good indicator of postoperative hypoparathyroidism. tient. In this prospective study we enrolled 159 consecutive patients who underwent total thyroidectomy with or without CND from January 2013 to March 2014.

We excluded patients who had abnormal serum calcium or intact PTH (iPTH), or chronic renal insufficiency, calcium metabolism disorder, history of malignancy of any other organ, or previous thyroid or neck surgery. We also excluded patients who underwent lobectomy or concurrent lateral neck dissection, or robotic or endoscopic thyroidectomy. Among the enrolled patients, we also excluded those who underwent parathyroid autotransplantation due to incidental parathyroidectomy or totally compromised vascularity of the parathyroid glands to eliminate the effect or bias from autotransplantation, and patients in whom less than 4 parathyroid glands were identified.

\section{Intraoperative assessment of viability of parathyroid glands}

All thyroidectomy was performed by three expert thyroid surgeons using $\times 2.5$ surgical loupes. A transverse low collar incision was used. We tried to identify and preserve all 4 parathyroid glands and their vascularity during the operation. The inferior thyroid artery was ligated at the level of its distal-most medial branches and the posterior branches of the superior thyroid artery were preserved, as far as possible, to minimize compromise of the blood supply to the parathyroid glands. We investigated whether the vascular pedicles of the parathyroid glands were intact and whether the color of the glands was normal or dusky, dark brown or black, or even pale at the end of thyroidectomy. When a parathyroid gland looked pale or of normal color even though its vascular pedicle seemed to be unsafe, we made a small incision in it with a cold knife to evaluate the blood supply to the gland. We scored the viability of each parathyroid gland according to its gross appearance and vascularity on a scale of 0 (normal), 1 (mildly compromised viability), 2 (moderately compromised viability), or 3 (severely compromised viability) (Table 1, Fig. 1) and recorded it on the operation record. The index of parathyroid viability score (IPVS) was defined as the sum of the viability scores of the 4 parathyroid glands.

\section{Analysis of parathyroid hormone and calcium}

Intact PTH was estimated by an electrochemiluminescence immunoassay (ELICA) on an Elecsys immunoassay analyzer (Roche, Indianapolis, IN, USA). Serum calcium, ionized calcium and $\mathrm{PPTH}$ were measured preoperatively and 1 day, 6 days, 1 month, and 6 months postoperatively, as well as whenever necessary. The reduction ratio of $\mathrm{PTTH}$ was calculated as (preoperative $\mathrm{iPTH}$ - postoperative iPTH) / preoperative iPTH $\times 100$.

Transient hypoparathyroidism was defined as any drop in serum iPTH below the normal limit $(15 \mathrm{pg} / \mathrm{mL})$ regardless of hypocalcemic symptoms, and permanent hypoparathyroidism was defined as lack of recovery of $\mathrm{PTTH}$ to within the normal range within 6 months. All patients were followed up for at least 6 months. 
Table 1. The scoring of parathyroid gland viability

\begin{tabular}{ll}
\hline Score & Finding \\
\hline 0 & Normal: parathyroid gland is of normal color with grossly intact vascular pedicle. \\
1 & Mleeding from an incision site in a gland. \\
2 & Moderately compromised viability: the parathyroid gland is discolored and dark brown or there is minimal bleeding from an incision site in a \\
gland. & Severely compromised viability: the parathyroid gland is discolored and dark brown to black or pale, or there is no bleeding from an incision \\
& site in a gland.
\end{tabular}
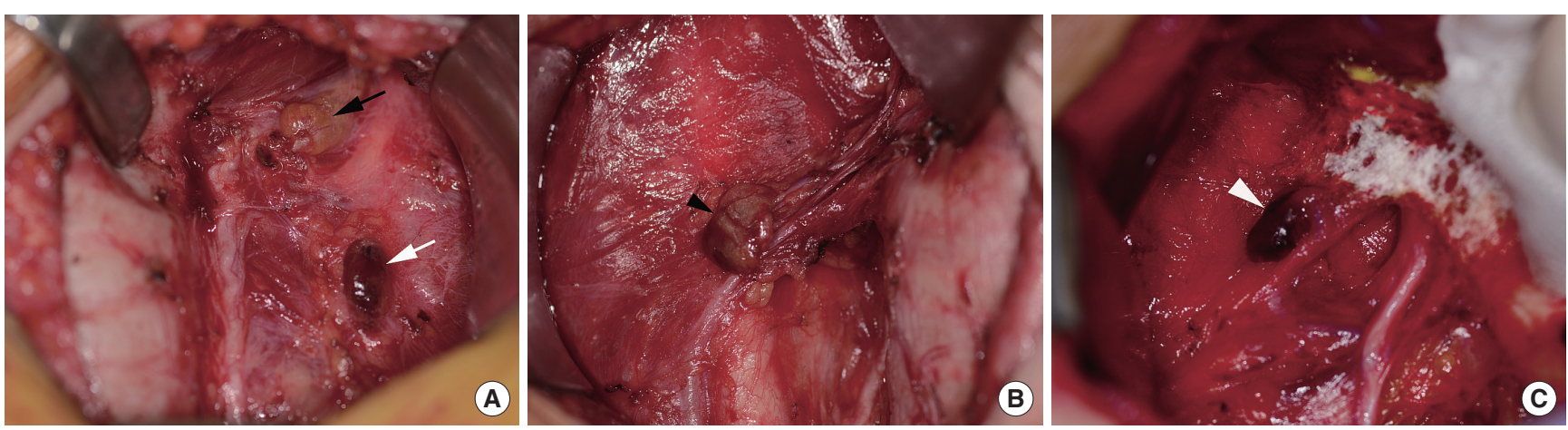

Fig. 1. Scoring of parathyroid gland viability according to intraoperative gross appearance and vascularity. (A) Black arrow indicates normal parathyroid with score 0 , and white arrow indicates moderately compromised parathyroid with score 2. (B) Black arrow head indicates mildly compromised parathyroid with score 1. (C) White arrow head indicates severely compromised parathyroid with score 3.

\section{Data analysis}

The patients were classified into normal, transient and permanent hypoparathyroidism groups according to postoperative iPTH function. IPVS were compared between the three groups using the Mann-Whitney $U$-test with SPSS ver. 18.0 (SPSS Inc., Chicago, IL, USA). A $P$-value of 0.05 or less was considered statistically significant. Postoperative parathyroid function and the reduction ratio of iPTH according to IPVS were compared on postoperative days 1 and 6 , and after 1 month and 6 months. The correlation between number of normal parathyroid glands and postoperative parathyroid function was also determined.

\section{RESULTS}

Of the 159 patients enrolled, 19 who underwent autotransplantation of parathyroid glands into the sternocleidomastoid muscle during the operation due to inadvertently removed or totally compromised parathyroid glands were excluded, as well as 29 patients in whom all four parathyroid glands could not be identified during the operation, or in whom an inadvertently removed parathyroid gland was noted after the operation. Finally, 111 patients were analyzed.

The characteristics of patients and tumors are summarized in Table 2. Of the 111 patients, 79 were women and 32 men, and the mean age was $52 \pm 13$ years. In terms of pathologic type, 107 patients (96.4\%) had papillary carcinoma and the other 4
Table 2. Clinicopathologic characteristics and complications

\begin{tabular}{lc}
\hline Characteristic & Value $(\mathrm{n}=111)$ \\
\hline Age $(\mathrm{yr})$ & $52 \pm 13$ \\
Gender & \\
Female & 79 \\
Male & 32 \\
Tumor size $(\mathrm{mm})$ & $14.7 \pm 14.3$ \\
Pathology & \\
Papillary carcinoma & $107(96.4)$ \\
Others & $4(3.6)$ \\
Extent of surgery & \\
Total thyroidectomy & $2(1.8)$ \\
Total thyroidectomy+ipsilateral CND & $39(35.1)$ \\
Total thyroidectomy+bilateral CND & $70(63.1)$ \\
Complication & \\
Transient vocal cord palsy & $2(1.8)$ \\
Permanent vocal cord palsy & 0 \\
Transient hypoparathyroidism & $25(22.5)$ \\
Permanent hypoparathyroidism & $4(3.6)$ \\
Hematoma & 0 \\
Seroma & $13(11.7)$ \\
\hline
\end{tabular}

Values are presented as number (\%) or mean \pm standard deviation. $\mathrm{CND}$, central neck dissection.

patients had follicular carcinoma, anaplastic carcinoma, Graves' disease and multinodular goiter, respectively.

A total of 109 patients (98.2\%) underwent CND. Ipsilateral and bilateral CND were performed in 39 patients $(35.1 \%)$ and 


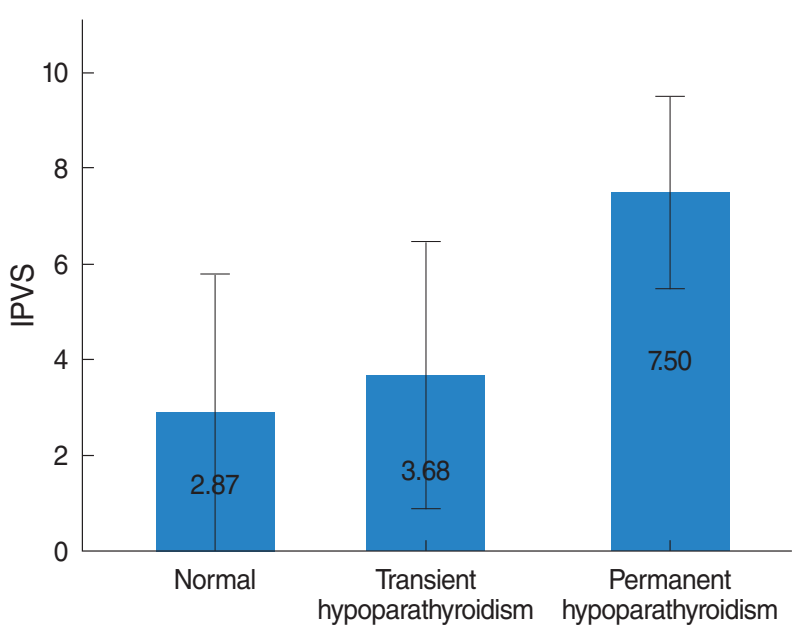

Fig. 2. Mean index of parathyroid viability score (IPVS) according to the postoperative parathyroid function. IPVS is significantly different in the three groups.
Table 3. Incidence of hypoparathyroidism according to the index of parathyroid viability score (IPVS)

\begin{tabular}{lcccc}
\hline & \multicolumn{3}{c}{ Postoperative parathyroid function } & \\
\cline { 2 - 3 } IPVS & Normal & $\begin{array}{c}\text { Transient } \\
\text { hypoparathyroidism }\end{array}$ & $\begin{array}{c}\text { Permanent } \\
\text { hypoparathyroidism }\end{array}$ \\
\hline 0 & $3(100)$ & 0 & 0 & $3(3)$ \\
1 & $10(91)$ & $1(9)$ & 0 & $11(10)$ \\
2 & $25(83)$ & $5(17)$ & 0 & $30(27)$ \\
3 & $17(74)$ & $6(26)$ & 0 & $23(21)$ \\
4 & $15(79)$ & $4(21)$ & 0 & $19(17)$ \\
5 & $8(53)$ & $7(47)$ & 0 & $15(14)$ \\
6 & $4(67)$ & $2(33)$ & 0 & $6(5)$ \\
7 & 0 & 0 & $3(100)$ & $3(3)$ \\
9 & 0 & 0 & $1(100)$ & $1(1)$ \\
Total & $82(74)$ & $25(23)$ & $4(4)$ & $111(100)$ \\
\hline
\end{tabular}

Values are presented as number (\%).

Table 4. The reduction ratio of intact parathyroid hormone according to the index of parathyroid viability score (IPVS)

\begin{tabular}{lcccc}
\hline IPVS & Postoperative 1 day (\%) & Postoperative 6 days (\%) & Postoperative 1 month (\%) & Postoperative 6 months (\%) \\
\hline 0-2 & $44.30 \pm 25.94$ & $41.08 \pm 36.42$ & $8.64 \pm 19.79$ & $25.20 \pm 25.20$ \\
$3-4$ & $57.83 \pm 26.43$ & $55.38 \pm 32.87$ & $30.35 \pm 25.66$ & $21.63 \pm 21.63$ \\
5-6 & $64.98 \pm 28.06$ & $64.62 \pm 34.04$ & $34.04 \pm 31.28$ & $23.78 \pm 23.78$ \\
7-9 & $91.05 \pm 6.76$ & $84.03 \pm 10.38$ & $74.65 \pm 5.44$ & $77.88 \pm 5.34$ \\
P-value for trend & 0.002 & 0.151 & 0.010 & $<0.001$ \\
\hline
\end{tabular}

Values are presented as mean \pm standard deviation.

70 patients $(63.1 \%)$, respectively. Transient vocal cord palsy occurred in 2 patients $(1.8 \%)$ but there was no permanent vocal cord palsy. Of the 111 patients, transient hypoparathyroidism occurred in 25 patients $(22.5 \%)$ and permanent hypoparathyroidism in 4 patients $(3.6 \%)$.

The IPVS of the three groups are shown in Fig. 2. The IPVS of the transient hypoparathyroidism group $(3.68 \pm 1.41)$ was higher than that of the normal group $(2.87 \pm 1.46 ; P=0.016)$. The IPVS was also higher in the permanent hypoparathyroidism groups $(7.50 \pm 1.00)$ than in the transient hypoparathyroidism group $(P<0.001)$.

Transient hypoparathyroidism did not occur when IPVS was 0. With increasing IPVS, the incidence of transient hypoparathyroidism increased; thus the frequencies of transient hypoparathyroidism were 1/11 (9\%) for IPVS 1, 5/30 (17\%) for IPVS 2, 6/23 (26\%) for IPVS 3, 4/19 (21\%) for IPVS 4, 7/15 (47\%) for IPVS 5 and $2 / 6$ (33\%) for IPVS 6 . There were no cases of permanent hypoparathyroidism when IPVS was 6 or less. However, when IPVS was 7 or more, all patients had permanent hypoparathyroidism (Table 3 ). When the IPVS were grouped into IPVS 0-2, $3-4$, and 5-6, the frequencies of transient hypoparathyroidism in the groups were $13.6 \%, 23.8 \%$, and $42.9 \%$ respectively.

The reduction ratio of iPTH increased significantly with increase of IPVS when measured on postoperative day 1 and after
1 and 6 months, but not when measured on postoperative day 6 (Table 4). The reduction ratio of iPTH decreased as parathyroid function was restored over time.

The relationship between postoperative parathyroid function and number of parathyroid glands of normal appearance and with intact vascular pedicles was evaluated. As the number of normal parathyroid gland decreased the incidence of transient hypoparathyroidism increased. There were 4 normal parathyroid glands in 3 patients, 3 in 14 patents, 2 in 37 patients, 1 in 37 patients and 0 in 20 patients. Transient hypoparathyroidism occurred in none of the 3 patients in the 4 normal parathyroid group, in $7 \%(1 / 14)$ of those with 3 normal glands, in 24\% (9/ 37 ) of those with 2 normal glands, in 19\% (7/37) of those with 1 normal gland, and in $40 \%(8 / 20)$ of those with no normal glands. When at least 1 parathyroid gland was normal, permanent hypoparathyroidism did not occur. However it occurred in $20 \%(4 / 20)$ of the patients with no normal viable parathyroid gland.

\section{DISCUSSION}

The risk of hypoparathyroidism increases with extent of thyroidectomy, concomitant central and/or lateral neck dissection, thy- 
roid cancer, Graves' disease and re-operation [18-20]. To decrease postoperative hypoparathyroidism, identification of all the parathyroid glands, and preservation of their vascular pedicles, are very important although it is not always possible to identify all four glands. Many surgeons advocate capsular or subcapsular dissection to avoid parathyroid injury. Nevertheless, injury to the parathyroid glands or their vascular pedicles cannot always be avoided. Also, despite meticulous dissection, unintentional parathyroidectomy does occur occasionally because the locations of the parathyroid glands are not constant and they can even be located within the thyroid capsule or thyroid parenchyma. The incidence of inadvertent parathyroid gland excision during thyroid surgery has been reported as $8 \%-21 \%$ [2-5].

Once hypoparathyroidism occurs, the prevention and treatment of hypocalcemic symptoms by prompt supplementation with calcium and/or vitamin $\mathrm{D}$ is important. There have been many attempts to assess the probability of good postoperative parathyroid function early postoperatively, and there are several reports that postoperative PTH level is a useful tool for predicting hypocalcemia, and that a decrease of PTH level either immediately or several hours postoperatively is associated with postoperative hypocalcemia $[12,13]$. The rapid PTH assay can virtually reflect real time parathyroid function because the halflife of PTH is as little as $2-5$ minutes [21]. Other authors have used serum calcium and ionized calcium to predict hypoparathyroidism. They reported that a gradual increase of calcium level within 24 hours postoperatively predicts normocalcemia $[14,15]$. However, intraoperative PTH assay is not available in all centers and it needs cost. Furthermore, operation time can be prolonged, because it needs time.

There are several reports of the correlation between postoperative hypocalcemia and the number of in situ preserved parathyroid glands. One study showed that the number of in situ preserved parathyroid glands was significantly lower in the permanent hypoparathyroidism group than the transient hypoparathyroidism group [8]. In our previous study we found that the incidence of transient and permanent hypoparathyroidism increased in proportion to the number of incidentally-removed parathyroid glands, and permanent hypoparathyroidism could be avoided if there was at least one parathyroid gland with an intact blood supply [17].

Although physical preservation of the parathyroid glands in situ is necessary, it does not ensure normal parathyroid function because the viability of the glands can be compromised by vascular injury. Some authors have reported that the number of identified parathyroid glands is not associated with the incidence of hypoparathyroidism [22], or even has a negative effect on it $[23,24]$. Many surgeons agree that parathyroid gland that seems to be nonviable should be taken out and transplanted $[6,11]$. Moreover, some surgeons advocated routine parathyroid autotransplantation to prevent permanent hypoparathyroidism $[25,26]$. However, in some cases of this study, we did not take out the parathyroid gland that seems to be comprimized mildly or moderately for autotransplantation if there was one or more other normal parathyroid glands based on the results of our previous study in that permanent hypoparathyroidism did not happen if there was at least one parathyroid gland with an intact blood supply [17].

Until now, there have been few attempts to evaluate the correlation between the viability of the parathyroid glands as assessed intraoperatively and postoperative parathyroid function, although surgeons traditionally assess the viability of the glands during the operation. There is one report that the function of discolored parathyroid glands recovered a short time after surgery [27], and the authors therefore insisted that autotransplantation of discolored parathyroid glands was not necessary. Our findings are quite different from those of the previous studies that evaluated the correlation between hypoparathyroidism and number of preserved parathyroid gland or the viability of discolored parathyroid glands itself. We found that it was possible to predict the probability of postoperative hypoparathyroidism quantitatively based on the viability of the parathyroid glands as determined by gross intraoperative findings and blood supply using a novel scoring system.

Traditionally, assessment of parathyroid gland viability has been based on the color change occurring in non-viable glands: a devascularized parathyroid gland was identifiable by its dusky or dark brown to black discoloration $[1,6,28]$. Also, several studies have shown that bleeding from an incision of a parathyroid gland is an important finding suggesting intact vascularity $[6,11,29,30]$. We have described a novel scoring system for parathyroid gland viability (IPVS) based on intraoperative findings developed in order to predict quantitatively postoperative parathyroid function, and IPVS proved to be significantly correlated with postoperative parathyroid function.

We found that there was no transient hypoparathyroidism when IPVS was 0 , and that the incidence of transient hypoparathyroidism increased with increasing IPVS. Interestingly, it increased roughly 2-fold with each 2-point increment of IPVS. Furthermore, there was no permanent hypoparathyroidism in patients whose IPVS was 6 or less. The number of normal parathyroid glands of normal appearance and with intact vascular pedicles was also correlated with postoperative parathyroid function. If the number of normal parathyroid gland was 4 , there was no hypoparathyroidism either transient or permanent. The incidence of transient hypoparathyroidism increased with decrease in the number of normal parathyroid glands. Surprisingly, there was no permanent hypoparathyroidism if at least one parathyroid gland was preserved. When there was no normal parathyroid gland, $40 \%(8 / 20)$ of the patients suffered transient hypoparathyroidism and $20 \%$ (4/20) permanent hypoparathyroidism.

This study has some limitations. First, assessment of the viability of parathyroid gland determined by intraoperative gross find- 
ings is somewhat subjective and there was also some interobserver variation. Second, some cases of arterial insufficiency with normal appearance may be missed because not all the parathyroid glands were examined by making small incisions in them. Third, IPVS cannot be applied to patients in whom all 4 parathyroid glands were not identified during thyroidectomy.

However, this quantitative scoring system might be helpful to predict the probability of permanent hypoparathyroidism after thyroidectomy and guide autotransplantation of parathyroid gland with compromised viability although surgeons have been judging the viability of the parathyroid gland during the operation empirically.

An objective method that measures the blood supply to the parathyroid glands accurately is needed to overcome the limitation of the subjective assessment by surgeons.

In conclusion, the IPVS appears to be a good quantitative indicator of the probability of hypoparathyroidism after thyroidectomy. The frequency of hypoparathyroidism increases with increasing IPVS, and permanent hypoparathyroidism does not occur when IPVS is 6 or less.

\section{CONFLICT OF INTEREST}

No potential conflict of interest relevant to this article was reported.

\section{REFERENCES}

1. Kahky MP, Weber RS. Complications of surgery of the thyroid and parathyroid glands. Surg Clin North Am. 1993 Apr;73(2):307-21.

2.Abboud B, Sleilaty G, Braidy C, Zeineddine S, Ghorra C,Abadjian G, et al. Careful examination of thyroid specimen intraoperatively to reduce incidence of inadvertent parathyroidectomy during thyroid surgery. Arch Otolaryngol Head Neck Surg. 2007 Nov;133(11): 1105-10.

3. Gourgiotis S, Moustafellos P, Dimopoulos N, Papaxoinis G, Baratsis S, Hadjiyannakis E. Inadvertent parathyroidectomy during thyroid surgery: the incidence of a complication of thyroidectomy. Langenbecks Arch Surg. 2006 Nov;391(6):557-60.

4. Lee NJ, Blakey JD, Bhuta S, Calcaterra TC. Unintentional parathyroidectomy during thyroidectomy. Laryngoscope. 1999 Aug;109(8): 1238-40.

5. Lin DT, Patel SG, Shaha AR, Singh B, Shah JP. Incidence of inadvertent parathyroid removal during thyroidectomy. Laryngoscope. 2002 Apr;112(4):608-11.

6. Lo CY, Lam KY. Postoperative hypocalcemia in patients who did or did not undergo parathyroid autotransplantation during thyroidectomy: a comparative study. Surgery. 1998 Dec;124(6):1081-6.

7. Ji YB, Lee DW, Song CM, Kim KR, Park CW, Tae K. Accuracy of intraoperative determination of central node metastasis by the surgeon in papillary thyroid carcinoma. Otolaryngol Head Neck Surg. 2014 Apr;150(4):542-7.

8. Pattou F, Combemale F, Fabre S, Carnaille B, Decoulx M, Wemeau $\mathrm{JL}$, et al. Hypocalcemia following thyroid surgery: incidence and prediction of outcome. World J Surg. 1998 Jul;22(7):718-24.
9. Ji YB, Lee KJ, Park YS, Hong SM, Paik SS, Tae K. Clinical efficacy of sentinel lymph node biopsy using methylene blue dye in clinically node-negative papillary thyroid carcinoma. Ann Surg Oncol. 2012 Jun;19(6):1868-73.

10. KuhelWI, Carew JF. Parathyroid biopsy to facilitate the preservation of functional parathyroid tissue during thyroidectomy. Head Neck. 1999 Aug;21(5):442-6.

11. Shaha AR, Jaffe BM. Parathyroid preservation during thyroid surgery. Am J Otolaryngol. 1998 Mar-Apr;19(2):113-7.

12. Chia SH, Weisman RA, Tieu D, Kelly C, Dillmann WH, Orloff LA. Prospective study of perioperative factors predicting hypocalcemia after thyroid and parathyroid surgery. Arch Otolaryngol Head Neck Surg. 2006 Jan;132(1):41-5.

13. Quiros RM, Pesce CE, Wilhelm SM, Djuricin G, Prinz RA. Intraoperative parathyroid hormone levels in thyroid surgery are predictive of postoperative hypoparathyroidism and need for vitamin D supplementation. Am J Surg. 2005 Mar;189(3):306-9.

14. Bentrem DJ, Rademaker A, Angelos P. Evaluation of serum calcium levels in predicting hypoparathyroidism after total/near-total thyroidectomy or parathyroidectomy. Am Surg. 2001 Mar;67(3):24951.

15. Luu Q, Andersen PE, Adams J, Wax MK, Cohen JI. The predictive value of perioperative calcium levels after thyroid/parathyroid surgery. Head Neck. 2002 Jan;24(1):63-7.

16. Lorente-Poch L, Sancho JJ, Ruiz S, Sitges-Serra A. Importance of in situ preservation of parathyroid glands during total thyroidectomy. Br J Surg. 2015 Mar;102(4):359-67.

17. Song CM, Jung JH, Ji YB, Min HJ, Ahn YH, Tae K. Relationship between hypoparathyroidism and the number of parathyroid glands preserved during thyroidectomy. World J Surg Oncol. 2014 Jul;12: 200.

18. Cavicchi O, Piccin O, Caliceti U, De Cataldis A, Pasquali R, Ceroni AR. Transient hypoparathyroidism following thyroidectomy: a prospective study and multivariate analysis of 604 consecutive patients. Otolaryngol Head Neck Surg. 2007 Oct;137(4):654-8.

19. Page C, Strunski V. Parathyroid risk in total thyroidectomy for bilateral, benign, multinodular goitre: report of 351 surgical cases. J Laryngol Otol. 2007 Mar;121(3):237-41.

20. Park CH, Song CM, Ji YB, Pyo JY, Yi KJ, Song YS, et al. Significance of the extracapsular spread of metastatic lymph nodes in papillary thyroid carcinoma. Clin Exp Otorhinolaryngol. 2015 Sep;8(3):28994.

21. Patel PC, Pellitteri PK, Patel NM, Fleetwood MK. Use of a rapid intraoperative parathyroid hormone assay in the surgical management of parathyroid disease. Arch Otolaryngol Head Neck Surg. 1998 May;124(5):559-62.

22. Hallgrimsson P, Nordenstrom E, Almquist M, Bergenfelz AO. Risk factors for medically treated hypocalcemia after surgery for Graves' disease: a Swedish multicenter study of 1,157 patients. World J Surg. 2012 Aug;36(8):1933-42.

23. Lang BH, Yih PC, Ng KK. A prospective evaluation of quick intraoperative parathyroid hormone assay at the time of skin closure in predicting clinically relevant hypocalcemia after thyroidectomy. World J Surg. 2012 Jun;36(6):1300-6.

24. Sheahan P, Mehanna R, Basheeth N, Murphy MS. Is systematic identification of all four parathyroid glands necessary during total thyroidectomy? a prospective study. Laryngoscope. 2013 Sep;123(9): 2324-8.

25. Lo CY, Lam KY. Routine parathyroid autotransplantation during thyroidectomy. Surgery. 2001 Mar;129(3):318-23.

26. Gauger PG, Reeve TS, Wilkinson M, Delbridge LW. Routine parathyroid autotransplantation during total thyroidectomy: the influence of technique. Eur J Surg. 2000 Aug;166(8):605-9.

27. Promberger R, Ott J, Kober F, Mikola B, Karik M, Freissmuth M, et 
al. Intra- and postoperative parathyroid hormone-kinetics do not advocate for autotransplantation of discolored parathyroid glands during thyroidectomy. Thyroid. 2010 Dec;20(12):1371-5.

28. Shaha AR, Burnett C, Jaffe BM. Parathyroid autotransplantation during thyroid surgery. J Surg Oncol. 1991 Jan;46(1):21-4.
29. Baumann DS, Wells SA Jr. Parathyroid autotransplantation. Surgery. 1993 Feb;113(2):130-3.

30. Schwartz AE, Friedman EW. Preservation of the parathyroid glands in total thyroidectomy. Surg Gynecol Obstet. 1987 Oct;165(4):32732. 